\title{
Management of neurogenic dysphagia
}

\author{
A M O Bakheit
}

Dysphagia is common in patients with neurological disorders. It may result from lesions in the central or peripheral nervous system as well as from diseases of muscle and disorders of the neuromuscular junction. Drugs that are commonly used in the management of neurological conditions may also precipitate or aggravate swallowing difficulties in some patients. Neurogenic dysphagia often results in serious complications, including pulmonary aspiration, dehydration, and malnutrition. These complications are usually preventable if the dysphagia is recognised early and managed appropriately.

\section{Physiological mechanisms of neurogenic dysphagia}

The act of swallowing may be viewed as three discrete but inter-related physiological stages: the oral, pharyngeal, and oesophageal phases. The oral phase is initiated voluntarily and serves to prepare the food bolus and deliver it to the pharynx. An adequately prepared and sufficiently large and cohesive food bolus triggers the swallow reflex by stimulating the sensory receptive field in the soft palate, dorsum of the tongue, epiglottis, and posterior pharyngeal wall. Simultaneously the larynx closes and the velum retracts upwards to prevent the entry of food and fluid into the nasal cavity. Coordination of respiration and swallowing is necessary to prevent the penetration of food into the airways. This is achieved by transient cessation of breathing, a process known as deglutition apnoea. The pharyngeal phase is followed by a prolonged expiration to avert mist aspiration, that is inhalation of air held in the pharynx (which is usually saturated with fluid and food particles). The swallow reflex triggers the oesophageal peristaltic movements and causes relaxation of the circopharyngeal sphincter. This, combined with the effects of gravity, facilitates the transmission of food down into the stomach.

Several factors contribute to the swallowing difficulties encountered in patients with neurogenic dysphagia. These include weakness of the oral musculature and tongue movements, failure to form a cohesive food bolus, reduced sensitivity of the pharyngeal receptors, and buccolingual apraxia. Although the abnormalities of swallowing associated with neurological disease predominantly affect the oropharyngeal phase, occasionally neurogenic dysphagia may result from disorders of innervation of the oesophagus.

\section{Stroke Unit, Mount Gould Hospital, Plymouth PL4 7QD, UK}

Correspondence to: Professor Bakheit

Submitted 23 February 2001 Accepted 2 July 2001

\section{Causes of neurogenic dysphagia}

Normal swallowing depends on the anatomical and functional integrity of numerous neural structures and extensive pathways in the central and peripheral nervous system. Lesions of the cerebral cortex, basal ganglia, brain stem, cerebellum, and lower cranial nerves may result in dysphagia. Degeneration of the myenteric ganglion cells in the oesophagus, muscle diseases and disorders of neuromuscular transmission, for example myasthenia gravis and Eaton-Lambers syndrome, are other less common causes.

\section{CEREBRAL CORTEX}

The commonest condition associated with dysphagia resulting from cortical lesions is stroke. Acute stroke is complicated by dysphagia in about $25 \%-42 \%$ of all cases. ${ }^{1}$ Dysphagia in these patients is usually associated with hemiplegia due to lesions of the brain stem or the involvement of one or both hemispheres. However, on rare occasions, dysphagia may be the sole manifestation of a cerebrovascular event. Dysphagia in the absence of other neurological symptoms and signs has been reported in patients with lacunar infarcts in the periventricular white matter ${ }^{2}$ and after discrete vascular brain stem lesions. ${ }^{3}$

Dysphagia in stroke is usually transient. Recovery of the swallowing ability occurs in almost $90 \%$ of cases within two weeks. However, the symptoms persist in about $8 \%$ of patients for six months or more. ${ }^{4}$ The occurrence of dysphagia in acute stroke does not appear to depend on the size or the site of the lesion. Interestingly, when it persists for a month or more after the stroke onset it is usually associated with right parietal lobe involvement.

\section{BASAL GANGLIA}

Dysphagia is usually a late feature in Parkinson's disease but is sometimes reported by patients in the early stages and may even be the presenting symptom in some cases. More than $80 \%$ of patients with Parkinson's disease have dysphagia but, as a rule, this is mild and has little or no effect on the patient's nutritional status. However, in about $10 \%$ of dysphagic parkinsonian patients the symptoms are severe and this generally correlates with the severity and duration of the disease. Tremor and speech disturbances have been found to be the main predictors of dysphagia in these patients.

The swallowing difficulties most frequently associated with Parkinson's disease relate to the oral phase (difficulties with lip closure and tongue movements) and the pharyngeal stage (complaints of food sticking in the throat). On videofluoroscopy these abnormalities are seen as abnormal bolus formation, multiple tongue elevations, delayed swallow reflex, and prolongation of the pharyngeal transit time with repetitive swallows to clear the throat. Drooling, which is commonly seen in patients with parkinsonism, is not due to excessive salivation but is an indication of bradykinesia of the 
oropharyngeal musculature. Other parkinsonian syndromes, for example Shy-Drager syndrome, multisystem atrophy, etc result in similar, but usually more severe symptoms.

Dysphagia is also common in spasmodic torticollis. In one study it was observed on videofluoroscopy in more than $50 \%$ of a randomly selected patients' sample. ${ }^{6}$ Interestingly, only two thirds of the study patients were symptomatic and the occurrence of dysphagia did not correlate with the patient's age or disease duration.

CEREBELLUM AND BRAIN STEM

Cerebellar disease and brain stem lesions resulting in bulbar and pseudobulbar palsy mainly affect the oral phase of swallowing. Poor coordination of the activity of the orofacial musculature may lead to inadequate lip seal, difficulties with the timing of the voluntary initiation of the swallow reflex, preparation of a cohesive food bolus, and delivery of the bolus to the pharynx.

PERIPHERAL NERVES AND MUSCLES

Isolated peripheral nerve lesions and degeneration of autonomic ganglion cells in the lower two thirds of the oesophagus (which results in achalasia) are rare causes of dysphagia. Achalasia is characterised by stasis of food and dilatation of the oesophagus due to reduced peristalsis and incomplete relaxation of the lower oesophageal sphincter. In addition to dysphagia, patients typically present with halitosis. The diagnosis is confirmed with endoscopy and studies of oesophageal motility.

Abnormalities of neuromuscular transmission, for example in myasthenia gravis, frequently cause difficulties with swallowing. In addition to dysphagia, patients with disorders of the neuromuscular junction often have dysphonia and dysarthria. Obvious weakness of oral and facial muscles may or may not be present. The diagnosis of the underlying disorder can usually be confirmed with single fibre electromyography.

DRUGS AND DYSPHAGIA

Many drugs may precipitate or aggravate swallowing difficulties. This effect is usually dose dependent and is often reversible with discontinuation of the drug. Sometimes reduction of the drug dose is sufficient. The mechanisms implicated in drug induced dysphagia are diverse. These include depression of the level of consciousness (sedatives and hypnotics), interference with the oropharyngeal phase of swallowing, a direct effect on brain stem neurones or blocking of acetylcholine release at the neuromuscular junction. Some drugs mediate their effect on swallowing by more than one mechanism.

Neuroleptic drugs delay the initiation of the swallow reflex, sometimes in the absence of obvious extrapyramidal features. On the other hand, dopaminergic agents may cause orofacial dyskinesia which affects the preparation of the food bolus and its delivery to the pharynx. Dysphagia due to poor preparation of the food bolus may also result from xerostomia caused by anticholinergic drugs. The benzodiazepines can adversely affect swallowing in at least two ways. Dysphagia in patients taking these drugs is usually associated with depressed level of consciousness but has also been reported in alert subjects. ${ }^{7}$ The latter effect could be explained by a direct inhibition of brain stem neurones that regulate swallowing. This is in keeping with observations from animal experiments. ${ }^{8}$

The most widely used drug that causes dysphagia due to inhibition of neural transmission at the neuromuscular junction is botulinum toxin type A. It is the drug of first choice for the treatment of spasmodic torticollis and may cause dysphagia in $10 \%-28 \%$ of these patients. This adverse effect is usually mild and transient, lasting 10-14 days. Clinical observations suggest that the incidence of dysphagia is increased when a large dose of the drug is injected (for example 750 units or more of botulinum toxin type A), especially if the dose is divided between multiple sites in the muscle.

Clinical manifestations of dysphagia and pulmonary aspiration

Patients with mild or moderately severe neurogenic dysphagia may not be aware of their swallowing difficulties. However, on direct questioning most of these patients would admit that they have been avoiding certain foods which they found difficult to chew or swallow. Weight loss may be the first presentation in some cases. Drooling occurs in dysphagic patients when they are sitting up and aspiration is common in the recumbent position, especially during sleep. Occasionally interrupted sleep may be the only indication of swallowing difficulties. Pain on swallowing (odynophagia) is not a symptom of neurogenic dysphagia and suggests the diagnosis of oesophagitis, usually secondary to candida infections. Nasal regurgitation of fluids occurs when palatal weakness is present.

\section{Assessment}

Assessment of swallowing function starts with a careful examination of the oral cavity. The presence of dysphagia and its severity can then be confirmed at the bedside by observing the patient during "trial swallows". Swallowing behaviour can be observed while the subject is taking food and fluids of different consistencies under "normal" everyday conditions. Coughing, splattering, and choking while eating are obvious signs. Change in the pattern of breathing or change in voice quality may also occur. Some patients attempt to compensate for their swallowing difficulties by taking small, frequent drinks during the meal in order to "wash down" the food bolus. Inspection of the oral cavity usually reveals pooling of secretions or food residue in the mouth.

In recent years a simple bedside measurement of the swallowing speed has been suggested as a diagnostic test for neurogenic dysphagia. ${ }^{9}$ The test, which has been shown to be specific and sensitive, consists of measuring the speed with which the patient drinks $150 \mathrm{ml}$ of cold tap water while sitting up. A swallowing 
speed of less than $10 \mathrm{ml} / \mathrm{s}$ suggests the presence of dysphagia. This test has obvious advantages over other methods of assessment such as videofluoroscopy and nasendoscopy, especially when regular and frequent monitoring of the patient's condition is necessary.

Videofluoroscopy is considered the gold standard for the evaluation of dysphagia. It permits the observation of the oral preparatory phase, the reflex initiation of swallowing, and the pharyngeal transit of the food bolus. However, it is not suitable for repeated assessments because of the undesirability of frequent exposure to radiation and the cost of the procedure. An alternative method is fibreoptic nasendoscopy. This procedure consists of introducing an endoscope through the nose into the nasopharynx and placing the tip just above the soft palate. The patient is then given food and drink coloured with a dye and before and after swallowing pharyngeal pooling is observed. Nasal endoscopy is a reliable method for assessing dysphagia and the risk of pulmonary aspiration but special expertise is necessary to carry out the procedure and to interpret its results.

Another useful method of bedside diagnosis of dysphagia and pulmonary aspiration is pulse oximetry. The use of this method is based on the premise that aspiration of food or fluid in the airways causes reflex bronchoconstriction that leads to ventilation-perfusion mismatch. The resulting oxygen desaturation of arterial blood can be readily measured with pulse oximetry. In a recent study pulse oximetry predicted aspiration or the lack of it in $81.5 \%$ of dysphagic stroke patients. ${ }^{10}$ This method is non-invasive, quick, and repeatable but its results must be interpreted with caution in smokers (high carboxyhaemoglobin concentrations may give false negative results) and in those with chronic lung disease.

Oesophageal manometry enables measurements of the intraoesophageal pressure gradient and is useful in the evaluation of dysfunction of the circopharyngeus muscle and abnormalities of oesophageal motility.

\section{Differential diagnosis}

A careful medical history and physical examination (supplemented by special investigations in some cases) are necessary to establish the cause of the swallowing difficulty and to exclude coincidental dysphagia due to obstructive lesions. In contrast to dysphagia due to oesophageal strictures or tumours, patients with neurogenic dysphagia find fluids more difficult to swallow than solids. This is probably because a solid (and more cohesive) food bolus is more likely to result in adequate pharyngeal stimulation and trigger a swallow reflex.

Dysphagia resulting from discrete brain stem lesions or confluent periventricular infarction may affect predominantly the volitional initiation of swallowing and spare reflexive deglutition. In these circumstances the patient's symptoms are often ascribed to globus hystericus. However, in the latter condition a "lump in the throat" is a characteristic complaint and the symptoms are usually associated with emotional distress. Patients with globus hystericus have a normal bolus transit time and seldom complain of difficulties with eating or drinking.

\section{Complications of dysphagia}

A serious complication of dysphagia is pulmonary aspiration. Severe swallowing difficulties, even for relatively short periods, may also lead to dehydration, reduced calorie intake, and malnutrition.

Pulmonary aspiration is defined as penetration of food or fluid into the airways below the true vocal cords. It may result in severe morbidity and sometimes mortality. About a third of patients with dysphagia aspirate food or fluid into their airways and in $40 \%$ of them aspiration is silent, that is does not trigger coughing or cause symptoms or signs of distress. Paradoxically, aspirating patients frequently do not complain of swallowing difficulties and there are no reliable clinical signs of this complication. Interestingly, a weak cough which is generally associated with the ability to protect the airways, is more likely to be present in aspirating rather than non aspirating dysphagic patients. ${ }^{11}$ Similarly, a diminished or absent gag reflex does not differentiate aspirating from non-aspirating patients. The detection of silent aspiration, therefore depends largely on a high index of clinical suspicion and may be confirmed by non-invasive bedside investigations such as pulse oximetry. ${ }^{10}$

\section{Management}

The aim of management is to prevent pulmonary aspiration, to maintain adequate food and fluid intake, and to correct nutritional deficiencies when present. Oral feeding has important social and psychological significance to patients and their families and should be continued whenever possible. In some patients oral intake is often not adequate even in the absence of significant swallowing difficulties. This may be due to excessive fatigue or cognitive impairment. In these patients oral food intake may be supplemented with gastrostomy tube feeding. It is preferable that such supplements are given at night. Withholding morning gastrostomy feeds usually stimulates the patient's appetite. An interdisciplinary team approach is essential for the optimal management of patients with neurogenic dysphagia. The core team should consist of a speech and language therapist, a dietitian, a nurse, and a physician.

Dysphagia in some patients may result from poor preparation of the food bolus because of ill fitting dentures or due to disease of the oral cavity, such as mouth ulcers or candida infections. These causes should be routinely looked for and treated. Care should be taken to avoid feeding when the patient is tired or distracted (for example while watching television). Talking while eating also increases the risk of aspiration and patients (and their carers) should be made aware of this. It is sometimes useful to plan the timing of meals so that they coincide with periods when the patient's functional abilities are maximal, for example, during the "on periods" in parkinsonian patients with "on/off” motor fluctuations. Frequent suction of copious saliva may be 
necessary in some cases. In dysphagic patients who have a tracheostomy, occlusion of the stoma with a speaking valve during swallowing reduces the risk of pulmonary aspiration presumably by normalising the pressure in the upper airways. The effects of posture on swallowing are well recognised. For example, "chin tuck" decreases the pharyngeal transit time of the food bolus, whereas "chin up" has the opposite effect. Head tilt to one side to maximise the effect of gravity on the unaffected side of pharynx is also a useful strategy on some occasions.

It has been shown that patients with weak tongue movements and those with poor pharyngeal clearance of the food bolus benefit from the use of gravity and posture to facilitate safe swallowing. Lying down on one side (at 45 degrees from flat) may be associated with less risk of aspiration than feeding in the upright position. ${ }^{12} 13$

REVIEW OF PATIENT'S MEDICATION

Sedative and other drugs that reduce the patient's level of arousal should be discontinued whenever possible. In patients with Parkinson's disease drug induced dyskinesia may cause or aggravate dysphagia and the successful management of this complication usually improves swallowing. Sometimes it is sufficient to avoid feeding during periods of peak dose dyskinesia. Drooling in parkinsonian patients is primarily due to swallowing difficulties rather than the excessive production of saliva. Anticholinergic drugs should be avoided in these cases as they aggravate dysphagia by increasing the viscosity of oral secretions. Viscid secretions interfere with bolus preparation and predispose to the formation of a mucous plug. Prescribing of benzodiazepines to dysphagic patients is not desirable and it is sensible that anticonvulsants are taken as a single dose at bedtime if possible.

DIETARY MODIFICATION

Maintenance of hydration and nutrition can be achieved safely in most patients with neurogenic dysphagia with dietary modification. Simple, but effective, measures include avoidance of dry and sticky food and eating food with uniform consistency. The use of starch based fluid thickeners, for example "Thick and Easy" and Vitaquick, is also an important management strategy. Tube feeding is usually required in only a minority of patients.

Patients with neurogenic dysphagia experience more difficulties with fluids than with solid food. This is probably due to the difficulty in controlling a thin bolus and/or a delay or absence of triggering the swallow reflex. The rationale for the use of fluid thickeners is that by increasing the viscosity of ingested fluids the resistance to flow of the bolus is increased. In addition, the duration of cricopharyngeal opening and the oropharyngeal transit time are increased. However, the optimal viscosity of fluids that ensures safe swallowing in patients with neurogenic dysphagia has not been established. In practice the required fluid thickness is judged subjectively and recorded using descriptive terms such as syrup or yoghurt consistency. This has the disadvantage that fluids with low viscosity may be served and result in pulmonary aspiration. On the other hand, fluids that are too thick are usually unpalatable and are often rejected by patients. The use of a viscometer for the preparation of the prescribed fluid thickness has been shown to improve the dietary management in these cases. $^{14}$

TUBE FEEDING

The direct delivery of nutrients into the stomach (or rarely into the jejunum) via a feeding tube is frequently used as the sole method of nutritional support of severely dysphagic patients who are at risk of pulmonary aspiration if fed orally. Tube feeding should be considered if the subject aspirates approximately $10 \%$ or more of the food bolus ( $M$ Collins, personal communication) or shows evidence of slow transit of the food bolus, that is more than 10 seconds, on videofluoroscopy. ${ }^{15}$ However, in some cases, for example when easy fatiguability makes swallowing unsafe, tube feeding can be used to supplement the daily oral intake. The patients will then be able to take their favourite foods orally and the rest of the calorie requirements will be given through the tube.

The use of a gastrostomy tube is preferred to naso-oesophageal intubation, especially when dysphagia is expected to be present for more than a few days. Nasogastric tube feeding is usually poorly tolerated and may make the patient irritable or even agitated. Extubation by patients is common and the volume of feeds delivered in this way is usually not adequate. In one study patients who were fed using a nasogastric tube received $55 \%$ of their feeds, whereas those fed with a gastrostomy tube had $93 \%$ of their prescribed daily intake. ${ }^{16}$ When nasogastric tube feeding is prescribed the use of fine bore tubes, for example Ryle's tube, is preferred to large bore ones. However, fine bore tubes are more likely to dislodge, kink, or block. They also deliver feeds at a relatively slow rate.

Some patients with neurological disease develop gastrointestinal ileus and in these patients enteral nutrition could be established with the intrajejunal administration of low residue solutions. Duodenal intubation is often facilitated by an intravenous injection of $20 \mathrm{mg}$ of metoclopramide a few minutes before the procedure. (Metoclpramide increases stomach peristalsis and relaxes the pyloric sphincter.)

Prolonged nasogastric tube feeding is not desirable. It often results in numerous complications including nasopharyngitis, oesophagitis, oesophageal strictures, epistaxis, pneumothorax, and nasopharyngeal oedema with associated otitis media. Furthermore, nasogastric tube feeding does not fully protect against aspiration and the association between nasogastric tube feeding and this complication is well documented. Ciocon et al have found that $43 \%$ of dysphagic patients aspirated in the first two weeks after nasogastric tube feeding was started. ${ }^{17}$ Elevation of the head of the bed during and for 1-2 hours after bolus nasogastric 
tube gastric and intrajejunal feeding reduces the risk of aspiration in these patients.

Feeding via a gastrostomy tube should be considered when dysphagia is likely to be progressive or to persist for long periods. For example, most clinicians would consider gastrostomy tube feeding in stroke patients if there are no signs of recovery of swallowing after the first week. However, there are no conclusive data to support this at present, but the results of a large ongoing multicentre study are eagerly awaited. ${ }^{18}$ In patients with motor neurone disease the option of percutaneous endoscopic gastrostomy (PEG) tube feeding should be offered early after the onset of dysphagia to supplement the oral intake and help maintain the muscle mass. Insertion of the feeding tube through a PEG, rather than a surgical gastrostomy, is a relatively simple, safe, and cost effective technique. PEG tube feeding is effective and is usually acceptable to patients and their carers. Transient, self limiting abdominal pain and diarrhoea may occur in the early postoperative period. Long term complications include tube obstruction and wound infection.

In some patients who are fed via a PEG tube pulmonary aspiration may occur and routine intrajejunal feeding has been suggested for these cases. However, technically it is easier to insert a gastric rather than an intrajejunal feeding tube. An additional advantage is that bolus gastric tube feeding is more physiological, particularly with respect to insulin secretion. Furthermore, because the feeds can be given intermittently it allows greater patient freedom (intrajejunal feeding should be given continuously rather than intermittently). Direct intrajejunal delivery of nutrients should probably be reserved for patients with gastro-oesophageal reflux, hiatus hernia, or recurrent aspiration on gastrostomy feeding.

Entral feeding can be started a few hours after the insertion of the PEG tube. The volume of feed is usually restricted in the first 24 hours to one litre and is given at a rate of 50 $\mathrm{ml} /$ hour. The volume of feed and the rate of its administration are then gradually increased over the following 3-4 days until the patient's daily nutritional requirements are met. Some clinicians prescribe "starter feeding regimens" in the first postoperative week. These are feeds diluted with sterile water. The concentration and osmolality are gradually increased over several days to full strength feeds. Starter regimens with clear water or diluted enteral solutions are thought to improve tolerance to feeds and reduce diarrhoea, but this has not been confirmed. In fact, a randomised controlled study demonstrated the poor relationship between osmolality of the feeds and the occurrence of diarrhoea in these patients. ${ }^{19}$ Furthermore, patients who were randomised to the starter regimen group received $20 \%$ less nutrients in the first seven days than the control subjects. Diarrhoea in patients on enteral feeding is usually due to treatment with antibiotics for coincidental infections, bacterial contamination of the feed and bolus feeding (the use of enteral feeding pumps to control the rate of feeding may prevent diarrhoea, nausea, and vomiting).

\section{SWALLOWING THERAPY}

A range of remedial therapies and training in the use of compensatory strategies may be helpful in the treatment of neurogenic dysphagia. These include exercises to strengthen the orofacial musculature, manoeuvres to improve poor laryngeal elevation and laryngeal closure during swallowing, and techniques to stimulate the swallow reflex. These methods are usually used before starting direct swallowing practice.

Exercises to enhance the function of the orofacial muscles are used to improve lip seal, mastication, and tongue movements. A simple technique known as "the supraglottic swallow" may improve the elevation and closure of the larynx during swallowing. During this manoeuvre the subject holds his/her breath and swallow and they release the air by coughing. Patients with delayed or absent swallow reflex often benefit from thermal stimulation of the oropharyngeal receptors. The procedure has been claimed to improve triggering of the swallowing action and to reduce the bolus transit time. It involves the repeated application of a small laryngeal mirror dipped in ice to the anterior faucial arch. Sensitisation may be repeated between swallows. Direct swallowing therapy can be started with small amounts of food (of the right consistency) under the supervision of a speech and language therapist when the risk of pulmonary aspiration is deemed to be low.

\section{SURGICAL TREATMENT OF NEUROGENIC} DYSPHAGIA

Cricopharyngeal myotomy has been shown to be an effective method of treatment of dysphagia in a number of neurological disorders including stroke, muscular dystrophy, and a significant proportion of patients with motor neurone disease. However, the careful selection of patients for this procedure is essential and two conditions must be satisfied. First, failure of relaxation of the pharyngeal sphincter must be demonstrated on videofluoroscopy. Secondly, the oral phase of swallowing, that is lip seal, voluntary initiation of swallowing, and the propulsive action of the tongue must also be preserved. Poor tongue movements (demonstrated on videofluoroscopy by the inability to propel or retrieve the food bolus) is a contraindication to cricopharyngeal myotomy. Patients with absent pharyngeal peristalsis or delayed triggering of the swallow reflex by 10 seconds or more are also unlikely to benefit from this treatment. Surgery for cricopharyngeal dysfunction after stroke and traumatic brain injury should be considered after the first three months of the disease onset.

Relaxation of the cricopharyngeus can also be achieved with "chemical cricopharyngeal myotomy" using botulinum toxin type A injections. ${ }^{20}$ The location of the cricopharyngeal muscle is determined with direct oesophagoscopy and electromyography (using a hooked 
wire electrode) and the toxin is injected transcutaneously into the dorsomedial part and into the ventrolateral party of the muscle on both sides. A total dose of botulinum toxin type A of $80-120$ units is usually sufficient and the mean beneficial effect of treatment is five months. The procedure usually requires a light general anaesthetic.

\section{Summary}

Dysphagia is a common impairment in patients with neurological disease and may be caused by lesions at any level of the neuroaxis. Frequently the swallowing difficulties are aggravated by sedatives and other drugs. The management, as well as the clinical outcome of dysphagia depend on the underlying pathology. In stroke, for example, most patients recover in the first month and only a small minority will require long term nutrition and hydration through a PEG feeding tube.

1 Kidd D, Lawson J, Nesbitt R, et al. Aspiration in acute stroke: a clinical study with videofluoroscopy. $Q f \mathrm{~F}$ Med 1993;86:825-9.

2 Celifarco A, Gerard G, Faegenburg D, et al. Dysphagia as the sole manifestation of bilateral strokes. Am $\mathcal{F}$ Gastroenterol 1990;85:610-13.

3 Buchholz DW. Clinically probable brain stem stroke presenting as dysphagia and nonvisualised by MRI. Dysphagia 1993;8:235-8.

4 Smithard DG, O'Neill PA, Martin DF, et al. The natural history of dysphagia following stroke. Dysphagia 1997;12: 188-93

5 Coates C, Bakheit AMO. Dysphagia in Parkinson's disease. Eur Neurol 1997;38:49-52.
6 Riski JE, Horner J, Nashold BS. Swallowing function in patients with spasmodic torticollis. Neurology 1990,40: patients

7 Wyllie E, Wyllie R, Cruse RP, et al. The mechanism of nitrazepam-induced drooling and aspiration. $N$ Engl f Med 1986,;14:35-8.

8 Hockman $\mathrm{CH}$, Bieger D. Inhibitory effect of diazepam on reflexly-induced deglutition. Proceedings of the Canadian Federation of Biological Sciences 1979;2:85.

9 Nathadwarawala KM, Nicklin J, Wiles CM. A timed test of swallowing capacity for neurological patients. $\mathcal{F}$ Neurol Neurosurg Psychiatry 1992;55:822-5.

10 Collins MJ, Bakheit AMO. Does pulse oximetry reliably detect aspiration in dysphagic stroke patients? Stroke 1997; 28: $1773-5$

11 Horner J, Massey W. Silent aspiration following stroke. Neurology 1988;38:317-19.

12 Ekberg O. Posture of the head and pharyngeal swallowing. Acta Radiol 1986;27:691-6.

13 Drake W, O'Donoghue S, Bartram C, et al. Eating in side lying facilitates rehabilitation in neurogenic dysphagia. Brain Inj 1997;11:137-42.

14 Goulding R, Bakheit AMO. Evaluation of the benefits of monitoring fluid thickness in the dietary management of monitoring fluid thickness in the dietary management

15 Park RHR, Allison MC, Lang J, et al. Randomised comparison of percutaneous endoscopic gastrostomy and nasogastric tube feeding in patients with persisting neurological dysphagia. BMF 1993;304:1406-9.

16 Wilson PS, Johnson AP, Bruce-Lockhart FJ. Videofluoroscopy in motor neurone disease prior to cricopharyngeal myotomy. Ann R Coll Surg Engl 1990;72:375-7.

17 Ciocon JO, Silverstone FA, Graver LM, et al. Tube feeding in elderly patients. Indications, benefits and complications. Arch Intern Med 1988;148:429-33.

18 The FOOD trial (Feed Or Ordinary Diet) - a multicentre trial to evaluate various feeding policies in patients admitted to hospital with a recent stroke. National Research Register 2001 , issue 2 .

19 Keohane PP, Attrill H, Love M, et al. Relation between osmolality of diet and gastrointestinal side effects in entral nutrition. BMF 1984;288:678-80.

20 Schneider I, Thumfart WF, Potoschnig C, et al. Treatment of dysfunction of the cricopharyngeal muscle with botulinum A toxin: introduction of a new non-invasive method. Ann Otol Rhinol Laryngol 1994;103:31-5. 